\title{
A Randomized, Double-blinded, Placebo- controlled Study to Evaluate the Efficacy and Safety of DA-9701 (Motilitone) in Patients With Constipation-type Irritable Bowel Syndrome and Functional Dyspepsia Overlap: A Pilot Study
}

\author{
Ju Yup Lee, ${ }^{1,2}$ Nayoung Kim, ${ }^{1,3 *}$ Hyuk Yoon, ${ }^{1}$ Cheol Min Shin, ${ }^{1}$ Young Soo Park, ${ }^{1}$ and Dong Ho Lee ${ }^{1,3}$ \\ ${ }^{I}$ Department of Internal Medicine, Seoul National University Bundang Hospital, Seoungnam, Gyeonggi-do, Korea; ${ }^{2}$ Department of Internal \\ Medicine, Keimyung University School of Medicine, Daegu, Korea; and ${ }^{3}$ Department of Internal Medicine and Liver Research Institute, Seoul \\ National University College of Medicine, Seoul, Korea
}

\section{Background/Aims}

To assess the effects and safety of DA-9701 (Motilitone) in patients with constipation-type irritable bowel syndrome (IBS-C) which frequently accompany functional dyspepsia (FD).

\section{Methods}

FD and IBS-C were diagnosed based on the Rome III criteria. Randomized subjects were administered $30 \mathrm{mg}$ of DA-9701 or placebo 3 times a day for 4 weeks. The study endpoints were evaluated the percentage of responders in the overall symptom evaluation of IBS-C and FD.

\section{Results}

Thirty IBS-C patients and 30 placebos were prospectively enrolled. The proportion of responders with improvement in overall symptoms of IBS-C was $53.33 \%$ in the DA-9701 group and $40.00 \%$ in the placebo group $(P=0.301)$. Compared to the placebo group, the decrease of abdominal pain score in the DA-9701 group was significantly higher at week 3 in the DA-9701 group (0.96 \pm 0.77 vs $0.55 \pm 0.79, P=0.042$ ) but no significance at week 4 . There was no significant difference in total IBS quality of life score at week 4 between the 2 groups $(P=0.897)$. Among patients with IBS-C accompanied by FD, the proportion of responders in the DA9701 group was $50.00 \%$ (15/30), which was higher than $31.03 \%(9 / 29)$ of the placebo group $(P=0.138)$.

\section{Conclusions}

DA-9701 showed trend of treatment efficacy in patients with IBS-C and FD overlap including overall improvement, and safety, compared to placebo but without significance probably due to small numbers. It is suggested the need for a large-scale clinical trial to confirm this preliminary effect of DA-9701.

(J Neurogastroenterol Motil 2022;28:265-275)

Key Words

DA-9701; Constipation; Dyspepsia; Irritable bowel syndrome

Received: October 21, 2020 Revised: March 30, 2021 Accepted: May 17, 2021

@) This is an Open Access article distributed under the terms of the Creative Commons Attribution Non-Commercial License (http://creativecommons. org/licenses/by-nc/4.0) which permits unrestricted non-commercial use, distribution, and reproduction in any medium, provided the original work is properly cited.

${ }^{*}$ Correspondence: Nayoung Kim, MD, PhD Department of Internal Medicine, Seoul National University Bundang Hospital, 82, Gumi-ro 173beon-gil, Bundang-gu, Seongnam, Gyeonggi-do 13620, Korea Tel: +82-31-787-7008, Fax: +82-31-787-4051, E-mail: nayoungkim49@empas.com 


\section{Introduction}

Irritable bowel syndrome (IBS) is defined as abdominal pain or abdominal discomfort associated with changes in bowel habits. ${ }^{1}$ It is a very common disease in the Western countries, showing a prevalence rate of $7-10 \%$ of the total population, ${ }^{2}$ and a Korean study also showed a prevalence rate similar to that of the Western countries at $8.0-9.6 \% .^{3,4}$ Due to recurring symptoms, quality of life (QoL) is greatly reduced, ${ }^{5,6}$ and medical expenses are increasing. ${ }^{7,8}$ Therefore, it is very important to improve symptoms with appropriate treatment. However, there are many difficulties in treatment as the symptoms are diverse, and the pathophysiology are not accurately known. Abnormal intestinal motility, visceral hypersensitivity, and psychosocial factors are suggested as important pathophysiological mechanisms of IBS. ${ }^{9}$

Recently, as mechanisms and mediators related to the movement, sensory, and secretory functions of the intestine have been revealed in more detail, various neurotransmitters and therapeutic agents targeting their receptors have been developed. A typical example is drugs that target 5-hydroxytryptamine (5-HT, serotonin) receptors. Among various drugs targeting 5-HT receptors, $5-\mathrm{HT}_{4}$ receptor agonists promote release of neurotransmitters such as acetylcholine and calcitonin gene-related peptide involved in gastrointestinal (GI) motility. It is known to be helpful in treatment of patients with constipation-type IBS (IBS-C) by controlling intestinal afferent sensation and promoting GI movement and secretory function. ${ }^{10}$ On the other hand, psychosocial factors are also thought to cause IBS, and many studies have reported that anti-depressants are effective in IBS. ${ }^{11}$ For this reason, buspirone, a $5-\mathrm{HT}_{1 \mathrm{~A}}$ agonist mainly used as an anti-anxiety agent was considered as a treatment option for IBS, and there have been attempts to combine $5-\mathrm{HT}_{3}$ receptor antagonist with a $5-\mathrm{HT}_{1 \mathrm{~A}}$ receptor agonist to treat IBS. ${ }^{12}$ In other words, it was to gain anti-anxiety effects in the central nervous system through the $5-\mathrm{HT}_{1 \mathrm{~A}}$ receptor agonist and control the movement of the peripheral GI tract through the $5-\mathrm{HT}_{3}$ receptor antagonist to increase the therapeutic effects in IBS. Therefore, $5-\mathrm{HT}_{1 \mathrm{~A}}$ receptor agonist may be a treatment option for patients with IBS.

DA-9701 (Motilitone tablet; Dong-A ST, Seoul, Korea) is a medicine made using natural substances extracted from seeds of Pharbitis Semen and root of Corydalis Tuber, which is a medicinal herb. ${ }^{13-15}$ It was reported that DA-9701 has effects through high affinity for the $5-\mathrm{HT}_{4}$ receptor and $5-\mathrm{HT}_{1 \mathrm{~A}}$ receptor and that its effects are exerted through the action of both receptors. ${ }^{15}$ Therefore, considering the function of DA-9701 as an agonist of 5- $\mathrm{HT}_{4}$ and
$5-\mathrm{HT}_{1 \mathrm{~A}}$ receptor, it may promote motor and secretory functions, regulate intestinal afferent sensation, and bring anti-anxiety effects. Then, DA-9701 may be an option for patients with IBS-C. Furthermore, in an experiment with interstitial cells of Cajal (ICC) extracted from the small intestine of mice, DA-9701 was reported to induce pacemaker current of ICC, suggesting that DA-9701 may restore the reduced function of ICC. ${ }^{16}$ From this background we made a hypothesis that DA-9701 could be an useful in the treatment of IBS-C, even though the indication of DA-9701 is functional dyspepsia (FD).

Moreover, many studies have suggested that FD is accompanied by a high rate with IBS, and a recently published meta-analysis study reported that the prevalence of IBS in patients diagnosed with FD is significantly higher with a relative risk of 8.0 (95\% CI, 5.7411.16) compared to those patients without FD. ${ }^{17} \mathrm{~A}$ study conducted in Korea also showed that there is a correlation between FD and IBS with a relative risk of $4.4(95 \% \mathrm{CI}, 1.21-15.71) .{ }^{18}$ From this background the aim of this study is to evaluate the effects and safety of DA-9701 in patients with IBS-C which is frequently overlapped with FD.

\section{Materials and Methods}

\section{Study Design and Intervention}

After screening for patients who meet the inclusion/exclusion criteria, 1-week observation period during which no treatment was provided and drugs that may have affect the effects of the test drug were stopped at least 3 days before the observation period. After assessing the symptom score during the observation period, subjects who met the criteria for transition to the treatment period were randomly assigned to DA-9701 or placebo groups and received the respective intervention for 4 weeks. The clinical study plan and design of the observation and treatment periods were as follows.

\section{Patients}

\section{Inclusion criteria}

Patients with IBS-C who met all of the following criteria were enrolled. (1) men and women aged 20 years to 75 years, (2) IBS was defined by the Rome III diagnostic criteria ${ }^{1}$ as patients who had abdominal pain or discomfort accompanied by more than 2 symptoms [1] symptoms improve after defecating, [2] when symptoms are present, there is a change in the number of defecations, and [3] when symptoms are present, there are changes in stool shape or 
condition) for 3 days every month in the last 3 months and whose symptoms started at least 6 months ago. (3) Patients who had constipation based on the Rome III IBS subtype criteria (Bristol stool form scale 1-2 stool form is $25 \%$ or more and Bristol stool form scale 6-7 stool form is less than 25\%). (4) Patients who underwent the following tests according to the patient's age after the onset of IBS symptoms or within 5 years and had no organic diseases [1] under 50 years old at the time of screening: sigmoidoscopy or colonography; [2] over 50 years of age at the time of screening: full colonoscopy or colonography; and [3] even when the patient was under the age of 50 at the time of screening, full colonoscopy or colonography could be performed at the discretion of the investigator). After selecting patients who met the IBS-C diagnostic criteria, FD was diagnosed by conducting an FD questionnaire. FD was diagnosed based the Rome III criteria that had at least 1 of the following symptoms: bothersome postprandial fullness, early satiation, epigastric pain, and epigastric soreness. ${ }^{19}$

\section{Criteria for proceeding to the treatment period}

Patients who satisfied the above screening selection criteria performed necessary tests during the 2 weeks of screening, underwent a wash out period, and had 1-week of observation period. At the end of the observation period, those who met all the following criteria were able to proceed to the treatment period: (1) patients whose IBS patient diary has been recorded for more than 5 days during the observation period, (2) patients who had at least 2 incidences of abdominal pain or discomfort during the 1 week of observation period and had visual analogue scale $\geq 30 \mathrm{~mm}$ of average abdominal pain or discomfort for 1 week, (3) patients who did not have Bristol stool form scale 6-7 stool form during the observation period, and (4) those who defecated less than 3 times for the 1-week observation period. All of the enrolled subjects were underwent colonoscopy and there were no significant diseases such as colitis present except polyps.

\section{Exclusion criteria}

Patients with any of the following were not eligible to participate in the study: (1) intolerant or hypersensitive to clinical study drugs, (2) abnormal findings in colonoscopy or colonography (results within the last 5 years can be used), (3) the following conditions within 6 months of screening; esophagitis, gastritis, duodenitis or ulcer, gastroesophageal reflux disease, bleeding in the GI tract, stricture or obstruction of the GI tract, infectious diarrhea, inflammatory bowel disease (Crohn's disease, ulcerative colitis, sensitive enteritis, ischemic colitis, and etc), pancreatic insufficiency, biliary tract disease, bile absorption disorders, (4) uncontrolled diabetes, uncontrolled high blood pressure, and thyroid dysfunction, (5) psychotic disorders, (6) history of malignant tumors (except for GI cancer, participation was possible if it did not recur within 5 years after treatment), (7) anemia, glaucoma, enlarged prostate, and collapsed patients, (8) history of major GI surgery or GI surgery with complications, (9) liver or renal dysfunction, (10) lactose intolerance not controlled by diet, (11) pregnancy, lactation, or fertile with no intention to use contraception during the study period, (12) expected to take prohibited drugs during the study period, (13) history of drug or medication and alcohol abuse, and (14) participated in other clinical studies within 30 days of screening.

Written informed consent was obtained from all patients before participation. All of the patients were informed of the study aims and methods, as well as the possible side effects. The Institutional Review Board of Seoul National University Bundang Hospital approved the study protocol (IRB No. B-1211-177-004), and the study was approved by the Korean Food and Drug Administration (20130027676).

\section{Randomization and Treatment}

SAS version 9.2 (SAS Institute, Cary, NC, USA) was used by a statistical expert who had no direct relationship with the clinical trial institution to randomly allocate the patients into the treatment group and placebo group in a 1:1 ratio following the block randomization method. The statistical expert delivered the randomization code to the code manager before the start of the clinical trial, so that a randomization envelope could be distributed. To maintain blinded conditions, only a minimal number of persons who did not have a direct relationship with the clinical trial institution was asked to check the randomization table and code before the end of the study. A placebo that has the same appearance as DA-9701 was used in this study to maintain the blinded conditions. Therefore, since both the treatment and placebo groups received that looked identical, it was not possible to distinguish which group the subject was assigned to unless the randomization number was disclosed. Randomized subjects were administered $30 \mathrm{mg}$ of DA-9701 or 1 tablet of placebo 3 times a day before each meal for 4 weeks.

\section{Safety Assessment}

Safety profiles were assessed by using review of systems and physical examination at screening and at each outpatient clinic visit. Laboratory tests including a complete blood count, biochemistry and electrolytes were performed before and after treatment. 


\section{Quality of Life}

Each participant completed the IBS-QoL questionnaire initially developed by Patrick et al. ${ }^{20}$ The Korean version of this questionnaire has been cross-culturally validated and used in a subsequent study by Park et al. ${ }^{21,22}$ This scale includes 34 items, each with a 5 -point Likert-type response scale ranging from 1 to $5(1=$ not at all, $2=$ slightly, $3=$ moderately, $4=$ quite a bit, and $5=$ extremely or a great deal). The individual responses to the 34 items were summed and averaged for a total score and then transformed to a 0-100 scale for ease of interpretation, with higher scores indicating better IBS specific QoL. There are also 8 domain (dysphoria, interference with activities, body image, health worry, food avoidance, social reaction, sexual function, and relationships) scores, with the total score of each also summed and transformed to a 0-100 scale. If a patient responded with "not at all" for all 34 items, the overall score of IBS-QoL would be 100. If a patient responded with "slightly," a score of 4 , for all items, the overall IBS-QoL score would be 80 . Therefore, if a score was more than 80 , the subscale was not considered to be associated with bowel problems. Moderate to severe IBS had an approximate score of 60-65 by the IBS-QoL. ${ }^{23}$

\section{Study Endpoint}

Intention-to-treat (ITT) set was intended for those subjects who received the investigational drug after randomization and whose data on primary efficacy endpoints after baseline could be obtained. Per protocol (PP) set was intended for subjects who completed the clinical trial and did not have a major deviation in the procedure of the clinical trial protocol. Reasons for PP set exclusion included dropout, violation of inclusion/exclusion criteria, and use of contraindicated drugs. Endpoint was analysed using both ITT set and PP set, and the main analysis target group was ITT set safety was analyzed using the safety set.

The primary endpoint was evaluated in terms of the percentage of responders in the overall symptom evaluation of IBS-C (responders: those who answered "Yes/No" to the question "Did last week's treatment adequately improve your symptoms" and whose number of weeks in which symptoms were adequately improved in $75 \%$ of the total treatment period). The secondary endpoint was the quantity of changes in abdominal pain and discomfort, stool consistency, bowel movement at weeks 1, 2, 3, and 4 compared the baseline and the quantity of changes in IBS-QoL at week 4 compared to the baseline. In patients with FD, the secondary endpoint was the percentage of responders in the evaluation of overall symptoms of $\mathrm{FD}$, the quantity of changes in epigastric pain or soreness, early satiety, postprandial fullness after meals at weeks 1, 2, 3, and 4 compared to the baseline.

\section{Statistical Methods}

A pilot study comparing DA-9701 with placebo was first attempted as the basis for calculating the appropriate number of subjects in large-scale clinical trials using DA-9701. As the recommended number of subjects in pilot studies is at least 30 per group, this study also intended to include 30 subjects per group. ${ }^{24,25}$

Mean \pm standard deviation, and median value (minimum and maximum) were presented as continuous variables. Frequency and percentage were presented as categorical variables. Normality test was performed for all continuous variables, and 2 sample $t$ test, paired $t$ test or Wilcoxon's rank sum test, or Wilcoxon's signed rank test were performed depending on whether the normality assumption was satisfied. Categorical variables were analyzed by Pearson's chisquare test or Fisher's exact test, depending on whether $80 \%$ or more of the cells with an expected frequency of 5 or more were found. The significance level of the analysis was set to $5 \%$ and 2-sided tests were conducted. $P$-value of less than 0.05 was considered significant. All statistical analysis was performed using SAS version 9.2.

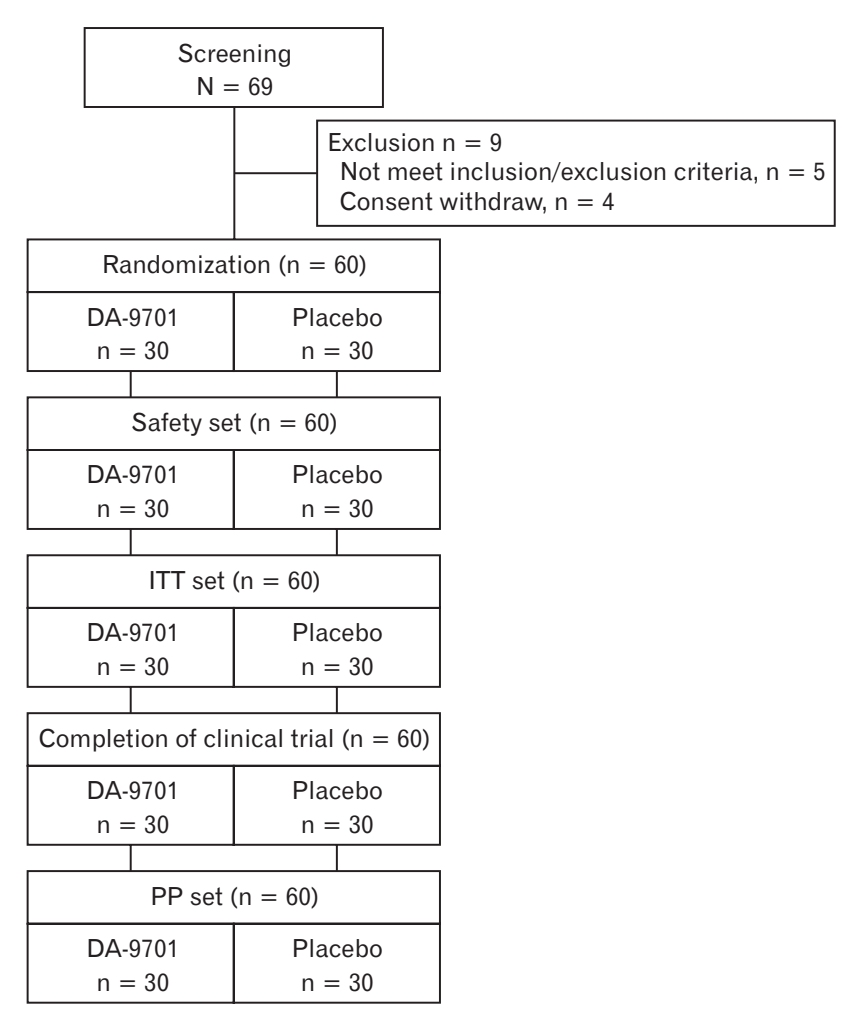

Figure 1. Subject disposition and analysis population of the study. ITT, intention-to-treat; PP set, per protocol set. 


\section{Results}

\section{Baseline Characteristics of the Study Subjects}

Among the 69 subjects who were screened, 60 subjects were enrolled in the study, excluding 5 and 4 subjects who did not meet the inclusion/exclusion criteria and withdrew their consent, respectively. All 60 subjects (30 subjects each in the DA-9701 group and the placebo group) who were randomly assigned were included in the safety set by receiving the investigational drug. In addition, all 60 subjects were included in the ITT set and PP set without dropouts or serious violation of the protocol (Fig. 1).

Analysis of demographic information and other basic informa-

Table. Baseline Characteristics of the Study Subjects

\begin{tabular}{|c|c|c|c|}
\hline \multirow{2}{*}{ Baseline characteristics } & DA-9701 & Placebo & \multirow{2}{*}{$P$-value } \\
\hline & $\mathrm{n}=30$ & $\mathrm{n}=30$ & \\
\hline Age (yr) & $47.97 \pm 14.71$ & $49.83 \pm 14.94$ & $0.836^{\mathrm{a}}$ \\
\hline $20-29$ & $2(6.67)$ & $5(16.67)$ & $0.285^{\mathrm{b}}$ \\
\hline $30-39$ & $10(33.33)$ & $3(10.00)$ & \\
\hline $40-49$ & $3(10.00)$ & $4(13.33)$ & \\
\hline $50-59$ & $7(23.33)$ & $10(33.33)$ & \\
\hline $60-69$ & $7(23.33)$ & $6(20.00)$ & \\
\hline Over 70 & $1(3.33)$ & $2(6.67)$ & \\
\hline \multicolumn{4}{|l|}{ Sex } \\
\hline Men & $8(26.67)$ & $5(16.67)$ & $0.347^{\mathrm{c}}$ \\
\hline Women & $22(73.33)$ & $25(83.33)$ & \\
\hline Height (cm) & $161.53 \pm 8.47$ & $159.57 \pm 6.29$ & $0.755^{\mathrm{a}}$ \\
\hline Weight (kg) & $58.56 \pm 11.25$ & $59.60 \pm 10.35$ & $0.455^{\mathrm{a}}$ \\
\hline $\operatorname{BMI}\left(\mathrm{kg} / \mathrm{m}^{2}\right)$ & $22.29 \pm 2.63$ & $23.33 \pm 3.27$ & $0.181^{\mathrm{d}}$ \\
\hline \multicolumn{4}{|l|}{ Smoking } \\
\hline \multicolumn{4}{|l|}{ History } \\
\hline Not identified & $0(0.00)$ & $0(0.00)$ & $0.418^{b}$ \\
\hline None & $26(86.67)$ & $29(96.67)$ & \\
\hline Past & $2(6.67)$ & $0(0.00)$ & \\
\hline Current & $2(6.67)$ & $1(3.33)$ & \\
\hline Amount & $0.75 \pm 0.35$ & $1.00 \mathrm{NA}$ & $1.000^{\mathrm{a}}$ \\
\hline \multicolumn{4}{|l|}{ Alcohol } \\
\hline \multicolumn{4}{|l|}{ History } \\
\hline Not identified & $0(0.00)$ & $0(0.00)$ & $1.000^{\mathrm{b}}$ \\
\hline None & $23(76.67)$ & $24(80.00)$ & \\
\hline Past & $1(3.33)$ & $0(0.00)$ & \\
\hline Current & $6(20.00)$ & $6(20.00)$ & \\
\hline Amount & $5.17 \pm 7.94$ & $13.00 \pm 9.88$ & $0.072^{\mathrm{a}}$ \\
\hline \multicolumn{4}{|l|}{ Concurrent FD } \\
\hline Yes & $30(100.00)$ & $29(96.67)$ & $1.000^{\mathrm{b}}$ \\
\hline No & $0(0.00)$ & $1(3.33)$ & \\
\hline IBS symptom duration (mo) & $42.83 \pm 35.29$ & $37.60 \pm 31.75$ & $0.298^{\mathrm{a}}$ \\
\hline Abdominal pain VAS score (mm) & $48.13 \pm 14.67$ & $47.13 \pm 7.52$ & $0.741^{\mathrm{d}}$ \\
\hline Abdominal discomfort VAS score $(\mathrm{mm})$ & $56.07 \pm 15.45$ & $50.57 \pm 8.64$ & $0.216^{\mathrm{a}}$ \\
\hline
\end{tabular}

${ }^{a}$ Wilcoxon's rank sum test.

${ }^{\mathrm{b}}$ Fisher's exact test.

'Pearson's chi-square test.

${ }^{\mathrm{d}}$ Two sample $t$ test.

BMI, body mass index; FD, functional dyspepsia; VAS, visual analogue scale.

Data are expressed as mean $\pm \mathrm{SD}$ or $\mathrm{n}(\%)$. 
tion of the ITT set participating in the study showed that the mean age of the DA-9701 group and the placebo group was $47.97 \pm$ 14.71 and $49.83 \pm 14.94$ years old, respectively (Table). In both groups, $73.33 \%$ (DA-9701 group, 22/30) and $83.33 \%$ (placebo group, 25/30) were women. The mean BMI was $22.29 \pm 2.63$ and $23.33 \pm 3.27 \mathrm{~kg} / \mathrm{m}^{2}$ in the DA-9701 group and placebo groups, respectively. There was also no significant difference between the 2 groups in smoking history and alcohol consumption. Assessment of FD in both groups showed that $100.00 \%$ (30/30) and $96.67 \%$ (29/30) of the subjects were in the DA-9701 and placebo group, respectively, had FD except 1 subject without FD in the placebo group (Table). The IBS symptom duration (DA-9701 group: $42.83 \pm 35.29$ months, placebo group: $37.60 \pm 31.75$ months), abdominal pain and abdominal discomfort score were not significant different between the 2 groups. In addition, there were no significant differences between the 2 groups in past disease, comorbid conditions, preceding drugs, concomitant drug, and medication compliance (Table).

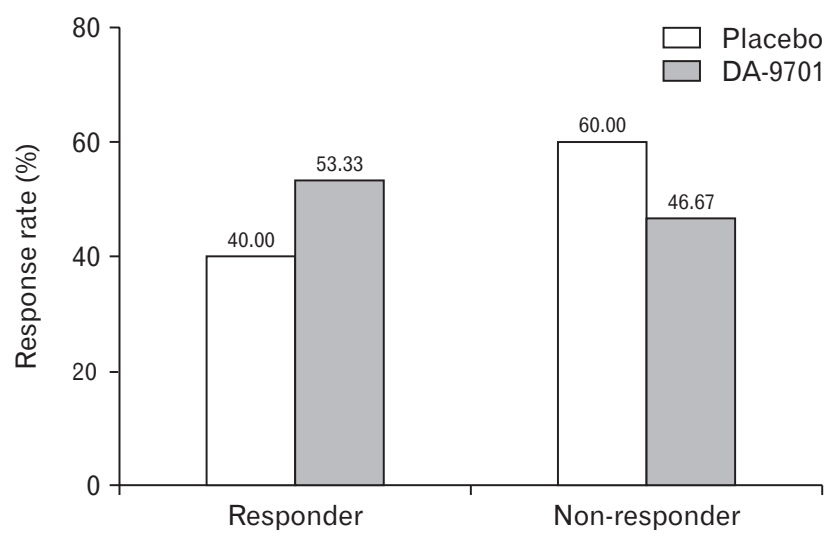

Figure 2. Evaluation of constipation-type irritable bowel syndrome (IBS-C) symptoms. The proportion of responders with improvement in overall symptoms of IBS-C was $53.33 \%$ in the DA-9701 group and $40.00 \%$ in the placebo group $(P=0.301)$. Responder, the patients whose number of weeks in which symptoms were adequately improved in $75 \%$ of the total treatment period.
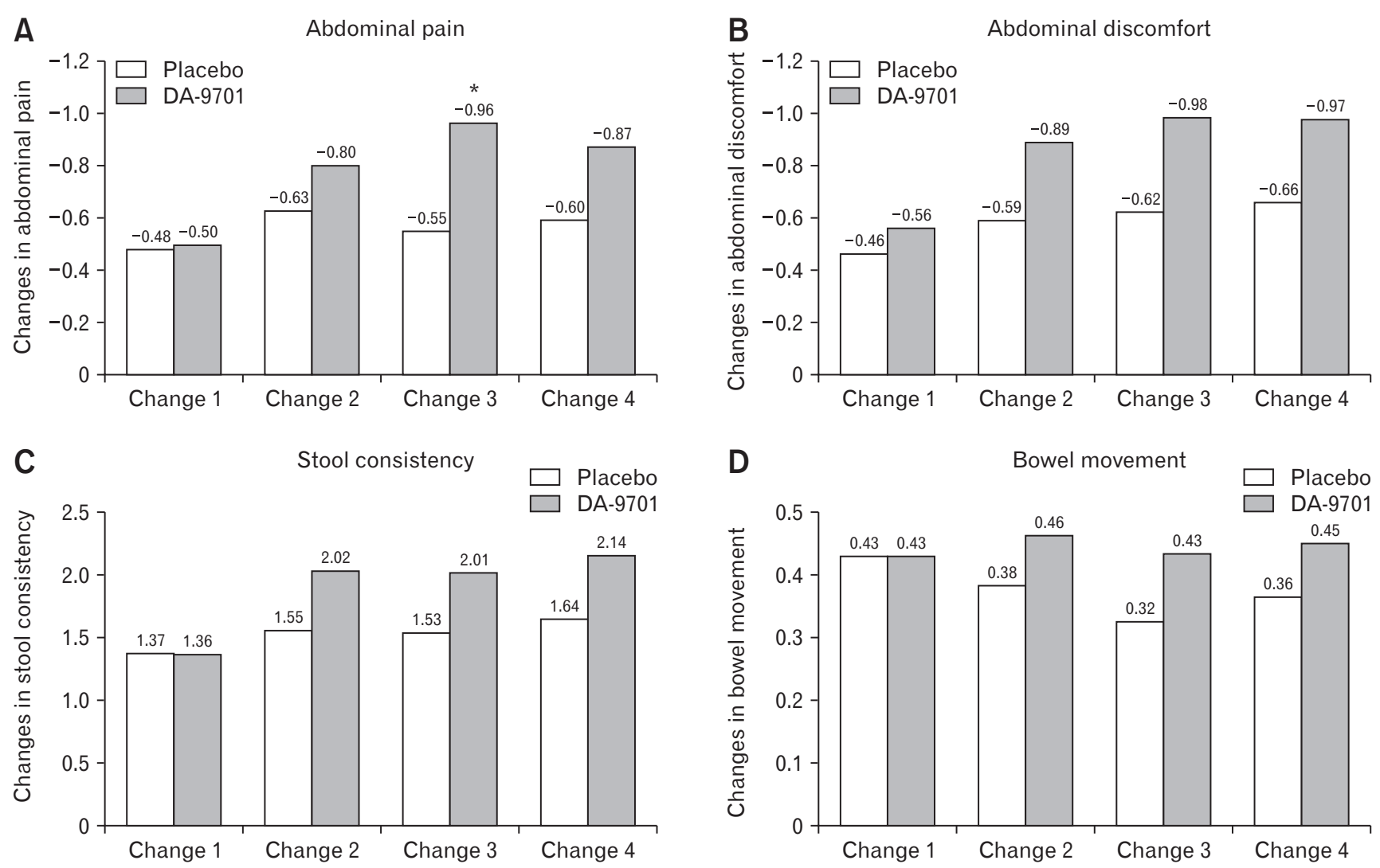

Figure 3. Changes in abdominal pain (A) and discomfort (B), stool consistency (C), and bowel movement (D) at weeks 1, 2, 3, and 4 compared to the baseline. Compared to the placebo group, the decrease abdominal pain score in the DA-9701 group tended to be higher, and the decrease at week 3 was significantly higher in the DA-9701 group $(P=0.042)$. Change $1=1$ week - baseline; Change $2=2$ weeks - baseline; Change $3=$ 3 weeks - baseline; Change $4=4$ weeks - baseline. ${ }^{*} P<0.05$. 


\section{Evaluation of Improvement in Overall Symptom of Constipation-type Irritable Bowel Syndrome}

The proportion of responders with appropriate improvement in overall symptoms of IBS-C was $53.33 \%$ in the DA-9701 group $(16 / 30)$ and $40.00 \%$ in the placebo group (12/30). Although the difference did not reach statistical significance the response rate was higher in the DA-9701 group than in the placebo group $(P=0.301)$ (Fig. 2).

\section{Changes in Abdominal Pain and Abdominal Discomfort, Stool Consistency, and Bowel Movement}

Comparison of abdominal pain reduction results showed DA9701/placebo group $0.50 \pm 0.87 / 0.48 \pm 0.79(P=0.951)$ at week 1 , DA-9701/placebo group $0.80 \pm 0.74 / 0.63 \pm 0.84(P=0.394)$ at week 2, DA-9701/placebo group $0.96 \pm 0.77 / 0.55 \pm 0.79$ $(P=0.042)$ at week 3 , and week 4 DA-9701/placebo group $0.87 \pm$ $0.77 / 0.60 \pm 0.76(P=0.161)$ at week 3 (Fig. 3A). Compared to the placebo group, the decrease in the DA-9701 group tended to be higher, and the decrease at week 3 was significantly higher in the DA-9701 group (Fig. 3A).

Abdominal discomfort gradually decreased until the fourth week of administration of the investigational drug, and the decrease in the DA-9701 group and the placebo group at week 4 was $0.97 \pm$ 0.88 and $0.66 \pm 0.87$ points, respectively (Fig. 3B). Although the differences between the 2 groups were not significant, the decrease in the DA-9701 group was higher than that of the placebo group $(P=0.167)$ (Fig. 3B)

Changes in the stool consistency during the administration of the investigational drug were compared to the baseline in the DA-
9701 and placebo groups. Up to week 4 of administration of the investigational drug, the stool consistency gradually became thinner, and the decrease in the DA-9701 and placebo groups at week 4 was $2.14 \pm 1.38$ points and $1.64 \pm 1.94$ points, respectively. Although the difference did not reach statistical significance, the stool consistency of subjects receiving DA-9701 was thinner compared to subjects receiving placebo $(P=0.110)$ (Fig. 3C).

The change in the number of bowel movements up to week 4 of administration of the investigational drug was DA-9701/placebo: $0.43 \pm 0.41$ times $/ 0.43 \pm 0.57$ times $(P=0.6044)$ at week $1, \mathrm{DA}-$ 9701/placebo: $0.46 \pm 0.36$ times/0.38 \pm 0.42 times $(P=0.4264)$ at week 2, DA-9701/placebo: $0.43 \pm 0.30$ times/0.32 \pm 0.40 times $(P=0.2363)$ at week 3 , and DA-9701/placebo: $0.45 \pm 0.42$ times/0.36 \pm 0.45 times at week 4 . The differences were not significant; however, the increase in the number of bowel movements was higher in the DA-9701 group than in the placebo group $(P=$ 0.449) (Fig. 3D).

\section{Changes in Irritable Bowel Syndrome-Quality of Life}

As a result of comparing the total IBS-QoL score at week 4 of administration of the investigational drug to the baseline, the DA-9701 group decreased by $3.85 \pm 19.99$ points while the placebo group decreased by $4.53 \pm 20.77$ points without significance $(P=0.897)$. The quantity of changes in 8 domains of QoL showed that QoL was improved in 7 domains including dysphoria (DA-9701/placebo: $-5.10 \pm 25.15 / 5.31 \pm 22.52$, $P=0.876)$, body image (DA-9701/placebo: $-1.88 \pm 25.06 /$ $-5.63 \pm 28.49, P=0.5904)$, health worry (DA-9701/placebo: $0.56 \pm 30.32 /-3.61 \pm 30.46, P=0.597)$, food avoidance (DA9701/placebo: $-8.06 \pm 21.38 /-8.61 \pm 23.42, P=0.924)$, social

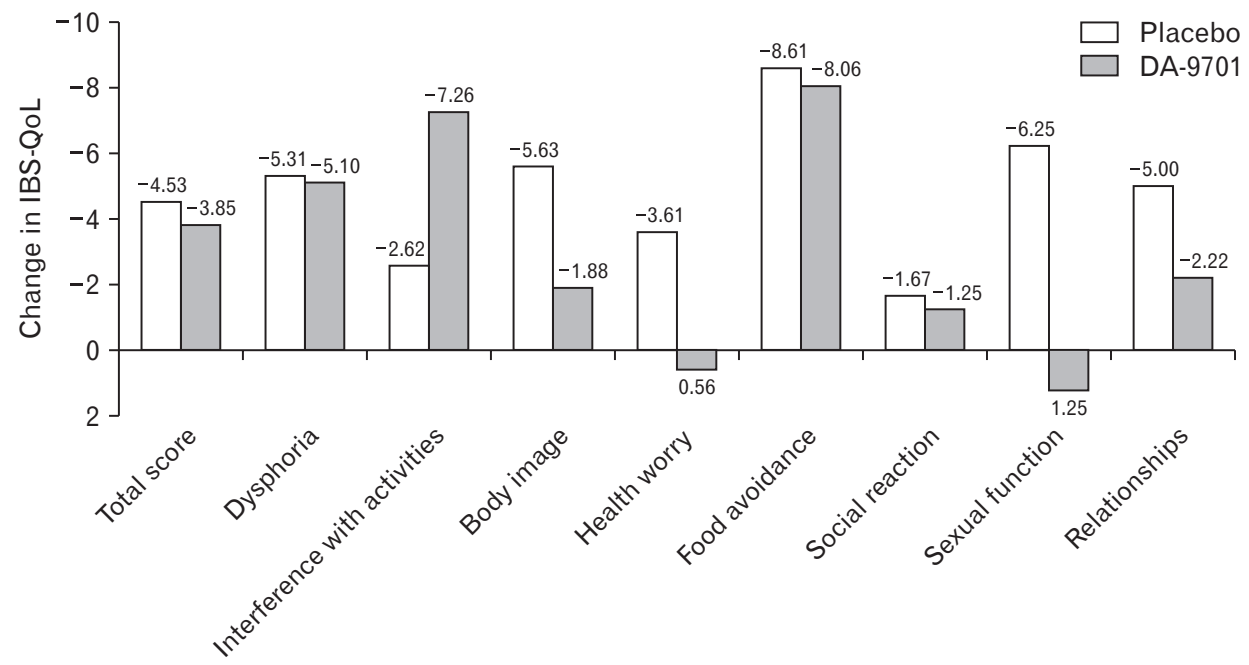

Figure 4. Changes in irritable bowel syndrome (IBS)-quality of life (QoL) at week 4 compared to the baseline. There was no significant difference in total IBS-QoL score at week 4 between the 2 groups $(P=0.897)$. 
reaction (DA-9701/placebo: $-1.25 \pm 23.18 /-1.67 \pm 25.05, P=$ 0.929), sexual function (DA-9701/placebo: $1.25 \pm 3.67 /-6.25 \pm$ $17.29, P=0.0981$ ), and relationships (DA-9701/placebo: $-2.22 \pm$ $23.97 /-5.00 \pm 20.83, P=0.818)$ in the placebo group compared to the DA-9701 group without significant differences. In interference with activities domain of $\mathrm{QoL}$, the quantity of change in the DA-9701 group was $-7.26 \pm 18.17$ points, which improved compared to $-2.62 \pm 20.42$ points in the placebo group $(P=0.356)$ without significant difference (Fig. 4).

\section{Subgroup Analysis of Functional Dyspepsia}

Among patients with IBS-C accompanied by FD, there were no significant differences between the 2 groups in the evaluation of overall symptoms of FD. However, the proportion of responders in the DA-9701 group was $50.00 \%$ (15/30), which was higher than $31.03 \%(9 / 29)$ in the placebo group $(P=0.138)$ (Fig. 5A). In addition, in terms of changes in the severity of symptoms including epigastric pain or soreness, early satiety, and postprandial fullness the improvement of symptoms was higher in the DA-9701

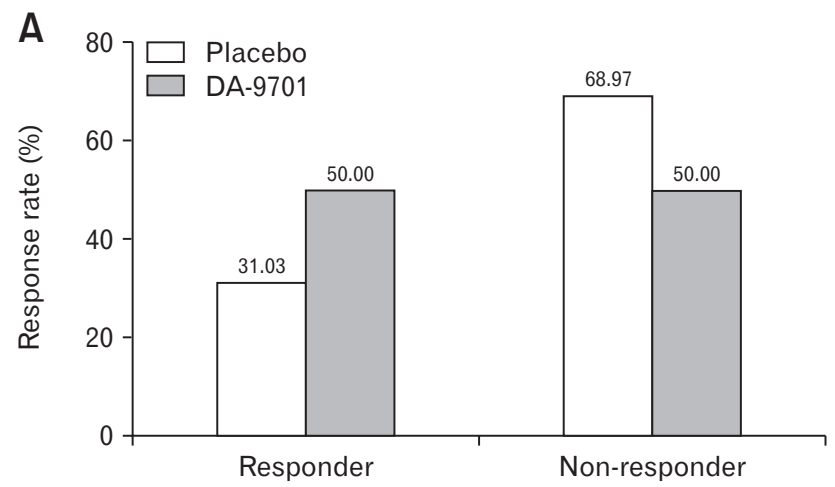

C

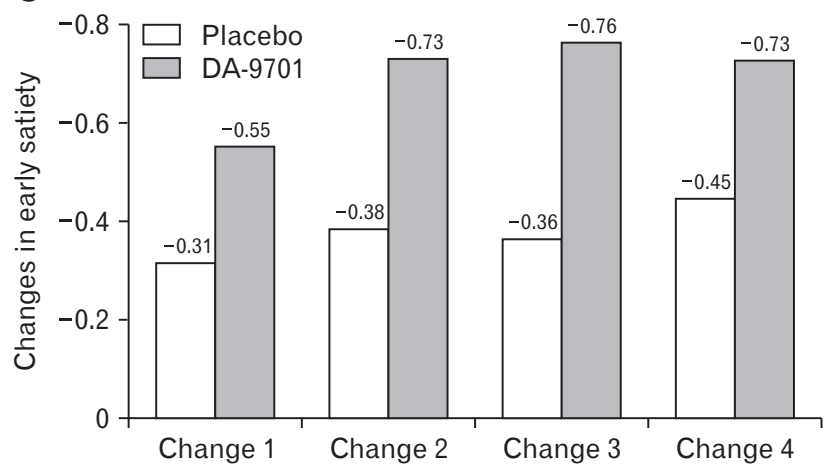

group than in the placebo group, but without significant difference between the 2 groups (the quantity of change at week 4 compared to baseline in epigastric pain or soreness, DA-9701: $-0.17 \pm 0.77$ points, placebo: $0.16 \pm 0.76$ points $[P=0.112]$; early satiety, $D A-$ 9701: $-0.73 \pm 0.89$ points, placebo: $-0.45 \pm 0.96$ points $[P=$ $0.261]$; and postprandial fullness, DA-9701: $-0.73 \pm 0.87$ points, placebo: $-0.34 \pm 1.16$ points, $[P=0.157]$ ) (Fig. 5B-D).

\section{Safety Assessment}

During the study period, 1 adverse reaction occurred before the administration of the investigational drug and the incidence rate of the treatment-emergent adverse event after administration of the investigational drug was $6.67 \%$ (2/30, 2 cases) in both the DA9701 group and the placebo group. Among these, the incidence rate of adverse drug reaction (ADR) was 3.33\% in both the DA-9701 group and the placebo group (1/30, 1 case), respectively (in the DA-9701 group, arthralgia was observed once in 1 subject while in the placebo group, somnolence was observed once in one subject). There were no serious adverse reactions or adverse reactions that
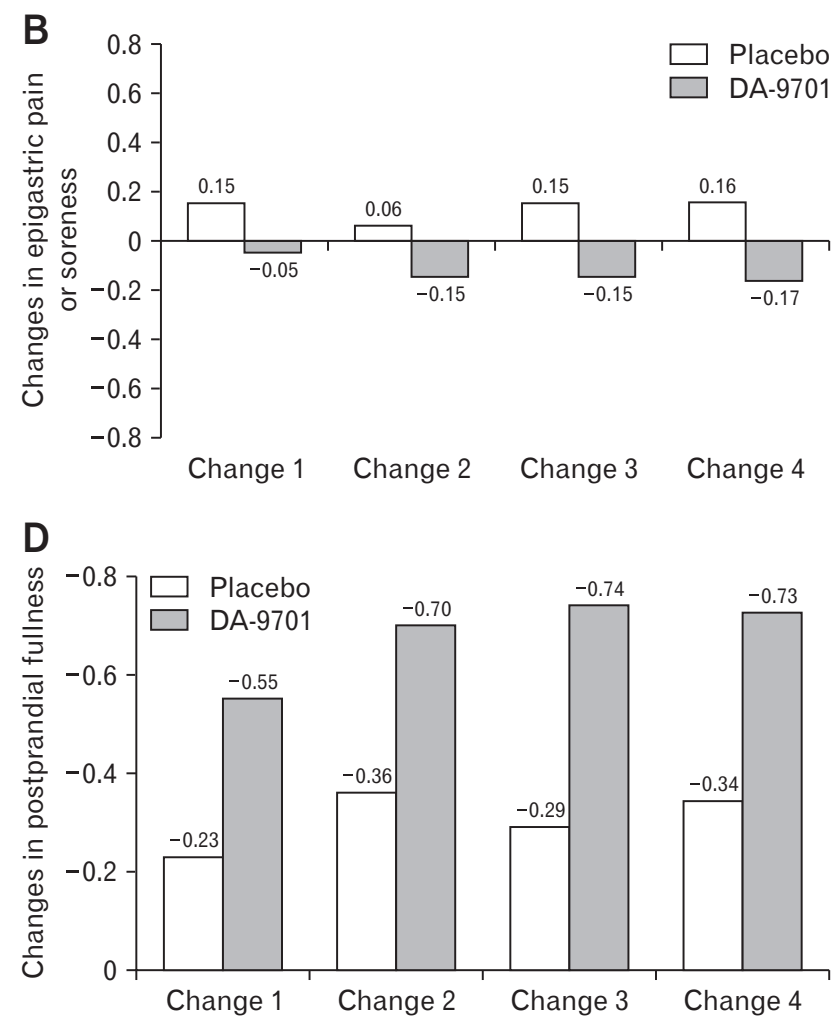

Figure 5. Subgroup analysis of functional dyspepsia. (A) The proportion of responders in the DA-9701 group was $50.00 \%$, which was higher than $31.03 \%$ in the placebo group $(P=0.138)$. (B-D) Changes in the severity of symptoms including epigastric pain or soreness, early satiety, and postprandial fullness were not significantly different between the 2 groups. Change $1=1$ week - baseline; Change $2=2$ weeks - baseline; Change 3 $=3$ weeks - baseline; Change $4=4$ weeks - baseline. 
led to withdrawal from the study. There were items with differences within the groups for the results of analysis of clinical laboratory, vital signs, and urine test results between the baseline and final visit. However, there were no changes that violated safety of the study, considering the clinical normal range, direction of changes, and amount of changes.

\section{Discussion}

This clinical trial study was conducted to evaluate the efficacy and safety of DA-9701 in patients with IBS-C and FD overlap by observing changes in the symptoms. The proportion of women was $73.33 \%$ and $83.33 \%$ in the DA-9701 group and placebo group, respectively, and the number of women were 3 to 5 times higher than the number of men, which was similar to the findings of previous studies. ${ }^{2,26}$ Characteristics such as demographic information and medical history of the 2 treatment groups were distributed in a comparatively equal manner between the 2 groups. Moreover, $100.00 \%$ (30/30) and 96.67\% (29/30) of subjects in the DA-9701 group and placebo group, respectively, also had FD, which was consistent with previous studies showing that many patients with IBS are accompanied by $\mathrm{FD}^{27-29}$

As a result of efficacy evaluation, there was no statistically significant difference in the proportion of responders with appropriate improvement in overall symptoms of IBS-C. However, the proportion of responders was higher with 53.33\% (16/30) in the DA-9701 group than in the placebo group with $40.00 \%$ (12/30). This finding is considered similar to a previous study comparing the effects of tegaserod in patients with IBS where the response rates of the DA9701 group and placebo group were $43.3-46.8 \%$ and $28.3-28.9 \%$, respectively. ${ }^{30,31}$ However, in the current study, no significant difference was found between the 2 treatment groups, mainly due to small number of enrollments, just 60 subjects. As this study was undertaken with a format of pilot study the enrollment was significantly less than 314 of the statistically considered minimum number of subjects. ${ }^{30}$

Moreover, the evaluation of symptoms of IBS-C (abdominal pain and abdominal discomfort, stool consistency, and number of bowl movements per day) showed slightly greater tendency of improvement than that of the placebo group as the period of DA-9701 administration increased. On the other hand, in IBS QoL survey, the DA-9701 group did not show a superior tendency compared to the placebo group except for interference with activities. It looks like that the symptom improvement observed in this study did not directly affect the QoL, and even in previous studies, symptom im- provement was not directly related to the improvement of QoL. ${ }^{32}$

When the response rate to the overall symptoms of FD and quantity of changes in individual symptoms (epigastric pain or soreness, early satiety, and postprandial fullness) were compared, significant differences were not observed. However, improvement effects in the DA-9701 group was higher than that in the placebo group. This is also thought to be caused by the lack of statistical power from the limited number of subjects, similar to the improvement in symptoms of IBS.

DA-9701 has an affinity for dopamine $\mathrm{D}_{2}, 5-\mathrm{HT}_{4}, 5-\mathrm{HT}_{1 \mathrm{~A}}$, and $5-\mathrm{HT}_{1 \mathrm{~B}}$ receptors. It brings antagonistic effects on the dopamine $\mathrm{D}_{2}$ receptor and agonistic effects on the $5-\mathrm{HT}_{4}, 5-\mathrm{HT}_{1} \mathrm{~A}$, and $5-\mathrm{HT}_{1} \mathrm{~B}$ receptors, resulting in various pharmacological effects. ${ }^{14,15,33,34}$ DA-9701 acts on the upper GI tract to improve gastric emptying and accommodation, helping to alleviate the symptoms of FD. ${ }^{15,35}$ In addition, DA-9701 also acts on the lower GI tract. For example, it has been reported that DA-9701 stimulates the ICC of murine small intestine, ${ }^{16}$ and it significantly increased the contraction and motility of the guinea pig GI tract and improved the lower GI tract transit in the opioid bowel dysfunction model. ${ }^{36}$ Clinical studies also showed the effects of DA-9701 on the lower GI tract. Kim et al reported that DA-9701 accelerated colon transit and improved symptoms in patients with functional constipation by measuring colonic transit time (CTT). ${ }^{37}$ In other words, in patients with constipation, CTT decreased by $32 \%$ after treatment with DA-9701 compared before treatment. ${ }^{37}$ This is consistent with the results of the study on prucalopride, a $5-\mathrm{HT}_{4}$ receptor agonist, where CTT decreased by $20-40 \% .^{38,39}$ Thus, these findings suggest that DA-9701 has effects on the $5-\mathrm{HT}_{4}$ receptor.

DA-9701 also improved stress-induced delay in gastric emptying and suppressed changes in plasma ACTH levels. ${ }^{40}$ Moreover, DA-9701 is also involved in the central corticotrophin-releasing factor pathway and was suggested to treat postoperative ileus. ${ }^{41}$ It improved GI transit delay in guinea pig experiments and reduced plasma ACTH levels and hypothalamic corticotrophin-releasing factor expression induced by abdominal surgery. ${ }^{41}$ Recently, several studies suggested that ICCs, which are distributed throughout the GI tract regulating GI smooth muscle movement by regularly emitting slow waves, may be involved in the pathophysiology of IBS. ${ }^{16,42}$ In patients with IBS-C the interval between the migratory motor complex cycles is long and it could be due to a decrease in the number of ICC which leads to a decrease in nitric oxide delivery. In addition, plasma concentration of 5-HT is decreased in patients with IBS-C. However, the $5-\mathrm{HT}_{2 \mathrm{~B}}$ receptor is expressed in ICC, and the activation of the $5-\mathrm{HT}_{2 \mathrm{~B}}$ receptor is known to mediate 
the proliferation of ICC. ${ }^{43,4+}$ Taken together, the activity of ICC is inhibited in patients with IBS-C, leading to decreased GI motility. ICCs are also thought to contribute to the pathophysiology of IBS by maintaining cholinergic neurotransmitters and controlling GI hormones and intestinal hypersensitivity. As in patients with IBS-C, decreased number or deterioration of the function of ICC is mostly observed. If the function of ICC is promoted through drugs such DA 9701 then it may contribute to improvement of symptoms.

In safety assessment, there were no significant differences in the incidence rate of adverse reactions and ADR between the DA9701 and placebo groups. In the DA-9701 group, increased blood creatine phosphokinase and arthralgia were observed once each (3.33\%) as adverse events, and arthralgia was reported as ADR. In particular, no adverse cardiovascular reactions such as myocardial infarction, stroke, and angina, which were the reasons for withdrawal of tegaserod, were not reported in DA-9701 that are 5- $\mathrm{HT}_{4}$ receptor and $5-\mathrm{HT}_{1 \mathrm{~A}}$ receptor agonist. Moreover, no changes leading to consideration of safety were observed in clinical laboratory tests (hematological, chemical, and urine test) and vital signs (blood pressure, pulse, and body temperature).

This study has several limitations. First, this is a pilot study conducted with 30 subjects per group. Pilot studies may have poor verification power due to the limited number of subjects. However, even though significant differences in treatment effects were not observed, it is thought that examining the treatment response rates between the DA-9701 group and placebo group allowed to evaluate whether a large-scale clinical trial would be feasible in the future, and also assess the approximate number of subjects required if such clinical trial studies are possible. Second, the 4-week treatment period can be short to calculate the exact sample size for the largescale clinical trial. However, in a previous study in which tegaserod, a $5-\mathrm{HT}_{4}$ receptor agonist, was administered to patients with IBS-C, the response rates of 4-week treatment in the tegaserod group and the placebo group were reported as $43.3 \%$ and $28.9 \%$, respectively. ${ }^{31}$ In other literatures, the primary treatment response rate was evaluated at 4 weeks. ${ }^{30,45}$ Therefore, We think that 4 weeks is adequate to observe the primary therapeutic effects of the drug.

In conclusion, this clinical study was a pilot study, and statistical significance verification was not possible due to the small number of subjects. However, it was possible to explore the clinical efficacy of DA-9701 in patients with IBS-C and FD overlap, including overall improvement, and safety, compared to placebo. In the future, evaluation of the efficacy and safety of DA-9701 through large-scale confirmatory clinical trials is necessary.
Financial support: This work was supported by the Seoul National University Bundang Hospital Research fund (Grant No. 062013-119).

\section{Conflicts of interest: None.}

Author contributions: Ju Yup Lee analyzed data and drafted the article; Nayoung Kim designed this study, collected the data, and supervised the writing of this manuscript; and Hyuk Yoon, Cheol Min Shin, Young Soo Park, and Dong Ho Lee supervised the writing of the manuscript. All authors have read and approved the final draft of this paper.

\section{References}

1. Longstreth GF, Thompson WG, Chey WD, Houghton LA, Mearin F, Spiller RC. Functional bowel disorders. Gastroenterology 2006;130:1480-1491.

2. Lovell RM, Ford AC. Global prevalence of and risk factors for irritable bowel syndrome: a meta-analysis. Clin Gastroenterol Hepatol 2012;10:712-721, e4.

3. Lee SY, Lee KJ, Kim SJ, Cho SW. Prevalence and risk factors for overlaps between gastroesophageal reflux disease, dyspepsia, and irritable bowel syndrome: a population-based study. Digestion 2009;79:196-201.

4. Park DW, Lee OY, Shim SG, et al. The differences in prevalence and sociodemographic characteristics of irritable bowel syndrome according to rome II and rome III. J Neurogastroenterol Motil 2010;16:186-193.

5. Rey E, García-Alonso MO, Moreno-Ortega M, Alvarez-Sanchez A, Diaz-Rubio M. Determinants of quality of life in irritable bowel syndrome. J Clin Gastroenterol 2008;42:1003-1009.

6. Gralnek IM, Hays RD, Kilbourne A, Naliboff B, Mayer EA. The impact of irritable bowel syndrome on health-related quality of life. Gastroenterology 2000;119:654-660.

7. Spiegel BM. The burden of IBS: looking at metrics. Curr Gastroenterol Rep 2009;11:265-269.

8. Camilleri M, Williams DE. Economic burden of irritable bowel syndrome. Proposed strategies to control expenditures. Pharmacoeconomics 2000;17:331-338.

9. Barbara G, Cremon C, Carini G, et al. The immune system in irritable bowel syndrome. J Neurogastroenterol Motil 2011;17:349-359.

10. Gershon MD, Tack J. The serotonin signaling system: from basic understanding to drug development for functional GI disorders. Gastroenterology 2007;132:397-414.

11. Ford AC, Talley NJ, Schoenfeld PS, Quigley EM, Moayyedi P. Efficacy of antidepressants and psychological therapies in irritable bowel syndrome: systematic review and meta-analysis. Gut 2009;58:367-378.

12. Asagarasu A, Matsui T, Hayashi H, Tamaoki S, Yamauchi Y, Sato M. Design and synthesis of piperazinylpyridine derivatives as novel 5-HT1A agonists/5-HT3 antagonists for the treatment of irritable bowel syndrome (IBS). Chem Pharm Bull (Tokyo) 2009;57:34-42. 
13. Lee TH, Son M, Kim SY. Effects of corydaline from corydalis tuber on gastric motor function in an animal model. Biol Pharm Bull 2010;33:958-962.

14. Lee TH, Choi JJ, Kim DH, et al. Gastroprokinetic effects of DA-9701, a new prokinetic agent formulated with pharbitis semen and corydalis tuber. Phytomedicine 2008;15:836-843.

15. Kwon YS, Son M. DA-9701: a new multi-acting drug for the treatment of functional dyspepsia. Biomol Ther (Seoul) 2013;21:181-189.

16. Choi S, Choi JJ, Jun JY, et al. Induction of pacemaker currents by DA9701, a prokinetic agent, in interstitial cells of cajal from murine small intestine. Mol Cells 2009;27:307-312.

17. Ford AC, Marwaha A, Lim A, Moayyedi P. Systematic review and meta-analysis of the prevalence of irritable bowel syndrome in individuals with dyspepsia. Clin Gastroenterol Hepatol 2010;8:401-409.

18. Park H. Functional gastrointestinal disorders and overlap syndrome in Korea. J Gastroenterol Hepatol 2011;26(suppl 3):12-14.

19. Tack J, Talley NJ, Camilleri M, et al. Functional gastroduodenal disorders. Gastroenterology 2006;130:1466-1479.

20. Patrick DL, Drossman DA, Frederick IO, DiCesare J, Puder KL. Quality of life in persons with irritable bowel syndrome: development and validation of a new measure. Dig Dis Sci 1998;43:400-411.

21. Park JM, Choi MG, Oh JH, et al. Cross-cultural validation of irritable bowel syndrome quality of life in Korea. Dig Dis Sci 2006;51:1478-1484.

22. Park JM, Choi MG, Kim YS, et al. Quality of life of patients with irritable bowel syndrome in Korea. Qual Life Res 2009;18:435-446.

23. Drossman D, Morris CB, Hu Y, et al. Characterization of health related quality of life (HRQOL) for patients with functional bowel disorder (FBD) and its response to treatment. Am J Gastroenterol 2007;102:1442-1453.

24. Lancaster GA, Dodd S, Williamson PR. Design and analysis of pilot studies: recommendations for good practice. J Eval Clin Pract 2004;10:307-312.

25. Browne RH. On the use of a pilot sample for sample size determination. Stat Med 1995;14:1933-1940.

26. Kim YS, Kim N. Sex-gender differences in irritable bowel syndrome. J Neurogastroenterol Motil 2018;24:544-558.

27. de Bortoli N, Tolone S, Frazzoni M, et al. Gastroesophageal reflux disease, functional dyspepsia and irritable bowel syndrome: common overlapping gastrointestinal disorders. Ann Gastroenterol 2018;31:639-648.

28. Lee SY, Ryu HS, Choi SC, Jang SH. Psychological factors influence the overlap syndrome in functional gastrointestinal disorder and quality of life among psychiatric patients in south Korea. Psychiatry Investig 2020;17:262-267.

29. von Wulffen M, Talley NJ, Hammer J, et al. Overlap of irritable bowel syndrome and functional dyspepsia in the clinical setting: prevalence and risk factors. Dig Dis Sci 2019;64:480-486.

30. Chey WD, Paré P, Viegas A, Ligozio G, Shetzline MA. Tegaserod for female patients suffering from IBS with mixed bowel habits or constipation: a randomized controlled trial. Am J Gastroenterol 2008;103:12171225.

31. Kellow J, Lee OY, Chang FY, et al. An Asia-Pacific, double blind, placebo controlled, randomised study to evaluate the efficacy, safety, and tolerability of tegaserod in patients with irritable bowel syndrome. Gut 2003;52:671-676.

32. Lembo AJ, Cremonini F, Meyers N, Hickling R. Clinical trial: renzapride treatment of women with irritable bowel syndrome and constipation - a double-blind, randomized, placebo-controlled, study. Aliment Pharmacol Ther 2010;31:979-990.

33. Kim ER, Min BH, Lee SO, Lee TH, Son M, Rhee PL. Effects of DA-9701, a novel prokinetic agent, on gastric accommodation in conscious dogs. J Gastroenterol Hepatol 2012;27:766-772.

34. Lee TH, Kim KH, Lee SO, Lee KR, Son M, Jin M. Tetrahydroberberine, an isoquinoline alkaloid isolated from corydalis tuber, enhances gastrointestinal motor function. J Pharmacol Exp Ther 2011;338:917924.

35. Min YW, Min BH, Kim S, Choi D, Rhee PL. Effect of DA-9701 on gastric motor function assessed by magnetic resonance imaging in healthy volunteers: a randomized, double-blind, placebo-controlled trial. PLoS One 2015;10:e0138927.

36. Hussain Z, Rhee KW, Lee YJ, Park H. The effect of DA-9701 in opioid-induced bowel dysfunction of guinea pig. J Neurogastroenterol Motil 2016;22:529-538.

37. Kim SY, Woo HS, Kim KO, et al. DA-9701 improves colonic transit time and symptoms in patients with functional constipation: a prospective study. J Gastroenterol Hepatol 2017;32:1943-1948.

38. Emmanuel A, Cools M, Vandeplassche L, Kerstens R. Prucalopride improves bowel function and colonic transit time in patients with chronic constipation: an integrated analysis. Am J Gastroenterol 2014;109:887894.

39. Cinca R, Chera D, Gruss HJ, Halphen M. Randomised clinical trial: macrogol/PEG 3350+electrolytes versus prucalopride in the treatment of chronic constipation -- a comparison in a controlled environment. Aliment Pharmacol Ther 2013;37:876-886.

40. Jung YS, Kim MY, Lee HS, Park SL, Lee KJ. Effect of DA-9701, a novel prokinetic agent, on stress-induced delayed gastric emptying and hormonal changes in rats. Neurogastroenterol Motil 2013;25:254-259, e166.

41. Jo SY, Hussain Z, Lee YJ, Park H. Corticotrophin-releasing factormediated effects of DA-9701 in postoperative ileus guinea pig model. Neurogastroenterol Motil 2018;30:e13385.

42. Yang BO, Zhou XC, Lan C. Impact of the alterations in the interstitial cells of cajal on intestinal motility in post-infection irritable bowel syndrome. Mol Med Rep 2015;11:2735-2740.

43. Borman RA, Tilford NS, Harmer DW, et al. $5-\mathrm{HT}_{2 \mathrm{~B}}$ receptors play a key role in mediating the excitatory effects of 5-HT in human colon in vitro. Br J Pharmacol 2002;135:1144-1151.

44. Wouters MM, Farrugia G, Schemann M. 5-HT receptors on interstitial cells of cajal, smooth muscle and enteric nerves. Neurogastroenterol Motil 2007;19(suppl 2):5-12.

45. Kim YS, Choi SC, Park JM, et al. The effect of tegaserod on symptoms and quality of life in Korean women with irritable bowel syndrome with constipation. J Neurogastroenterol Motil 2010;16:61-70. 